\title{
Extended Low Hartmann Operation with Total Mesorectal Excision - Optimal Surgical Treatment in Stage IV Mid and Upper Rectal Cancer
}

\author{
Timofeiov Sergiu ${ }^{1,3}$, Mihaela Elena Breabăn ${ }^{2}$, Drug Vasile ${ }^{1,5}$, Gervescu Andrei ${ }^{4}$, Huțanu I,4, and Scripcariu Viorel ${ }^{1,4}$ \\ ${ }^{1}$ University of Medicine and Pharmacy Gr.T. Popa, Faculty of Medicine, lasi, Romania \\ ${ }^{2}$ University Alexandru loan Cuza, Faculty of Computer Science, lasi, Romania \\ ${ }^{3}$ Third Surgical Clinic of Sf. Spiridon Hospital, Iasi, Romania \\ ${ }^{4}$ First Surgical Oncology Unit, Regional Institute of Oncology laşi, Romania \\ Instiute of Gastroenetrology and Hepatology Sf. Spiridon Hospital lasi, Romania
}

\begin{abstract}
Background: The main surgeries that can be performed in upper and mid rectal cancer are low anterior rectal resection (LAR), lateral colostomy (LC), and extended Hartmann operation with mesorectal excision (EHO). Compared to lateral colostomy, EHO is a curative surgery; it also takes a shorter time to perform and has a lower mortality compared with LAR.
\end{abstract}

Objective: The aim of this study was to analyze the postoperative surgical complications in patients who underwent LAR, LC, or EHO.

Methods: The study is a prospective analysis based on a Surveillance protocol for patients with liver metastases of colorectal origin used in First Surgical Oncology Unit, Regional Institute of Oncology laşi, Romania. The postoperative complications occurring within 60 days postoperatively were analyzed.

Results: In the interval June 2012 - May 2014, 87 patients were diagnosed with upper and mid-rectal cancer and liver metastases; LAR was performed in 18 patients, LC in 19 patients and in 50 patients EHO with pelvic drainage abdominally exteriorized or perineally ( 37 and 13 patients, respectively). The postoperative complications related to surgical wound, stoma, pelvic abscess, postoperative bleeding, prolonged postoperative ileus, anastomotic leakage, reinterventions, readmissions, and mortality were analyzed. A higher rate of pelvic abscesses was found in EHO patients $(24 \%, n=12)$, of which 9 patients $(18 \%)$ required reinterventions for drainnage of pelvic fluid collection; all these patients had pelvic drainage exteriorized abdominally. No presacral abscesses were recorded in patients with pelvic drainage exteriorized perineally.

Conclusions: Extended Hartmann Operation (EHO) remains a safe technique, that shares some indications with LAR and LC; however, it is associated with a higher percentage of pelvic abscesses and reinterventions that can be avoided by postoperative perineal drainage of the presacral space

Keywords: Extended Hartmann Operation; Rectal cancer; Pelvic abscess; Perineal drainage

\begin{abstract}
Abbreviation: LAR=Low Anterior Rectal Resection; LC=Lateral Colostomy; EHO=Extended Hartmann Operation with Mesorectal Excision; EHO-abd=Extended Hartmann Operation with Mesorectal Excision and Pelvic Drainage Abdominally Exteriorized; EHOper=Extended Hartmann Operation with Mesorectal Excision and Pelvic Drainage Perineally Exteriorized
\end{abstract}

\section{Introduction}

Colorectal cancer is the third most common cancer in the world with nearly 1.4 million new cases diagnosed in 2012 [1]. In Romania, the incidence has doubled in the last 20 years reaching 22.8 cases per 100,000 population, making it the second leading cause of cancer death after lung cancer [1].

Colorectal cancer treatment is multimodal, involving a multidisciplinary team and periodic reassessments of the patients to determine the need for adjusting the initial therapeutic strategy. In terms of finding an optimal therapeutic strategy, the most difficult challenge a surgeon faces is the advanced stages of rectal cancer. The presence of distant metastases (M1a, b) and/or a high degree of local invasion (T3, T4a, b) in patients with rectal cancer most often involves a new interpretation of existing treatment protocols, and their adjustment depending on age, associated diseases, anesthetic risk, and patient decision after he has been properly informed.
In the last three decades significant progress has been made in the management of rectal cancer by the introduction of the concept of total mesorectal excision [2], neoadjuvant radiochemotherapy [3,4], updating of TMN classification based on clinical studies, description of the two mechanisms of colorectal carcinogenesis ( $\mathrm{LOH}+$ phenotype and MSI + phenotype) [5,6], identification of new locoregional therapies, discovery of new chemotherapeutic molecules, and the introduction of targeted therapies with monoclonal antibodies. Due to these advances, the median survival of patients with metastatic rectal cancer may exceed 24 months $[7,8]$. Surgical removal of the primary tumor and metastases still remains the only curative treatment.

The main surgical options for upper and mid rectal cancer are:

*Corresponding author: Sergiu Timofeiov MD, University of Medicine and Pharmacy Gr.T. Popa, Faculty of Medicine, lasi, Romania, Tel. / Fax: 004023224 08 22; E-mail: stimof@yahoo.com

Received September 01, 2014; Accepted October 13, 2014; Published October 20, 2014

Citation: Timofeiov S, Breabăn ME, Drug V, Gervescu A, Huțanu I, et al. Extended Low Hartmann Operation with Total Mesorectal Excision - Optimal Surgical Treatment in Stage IV Mid and Upper Rectal Cancer. Journal of Surgery [Jurnalul de chirurgie] 2014; 10(3): 223-227 DOI: 10.7438/1584-9341-10-3-4

Copyright: (c 2014 Timofeiov S, et al. This is an open-access article distributed under the terms of the Creative Commons Attribution License, which permits unrestricted use, distribution, and reproduction in any medium, provided the original author and source are credited. 
- low anterior resection (LAR) with total mesorectal excision or mesorectal transection by classical or laparoscopic approach - the preferred treatment for rectal cancer.

- classic or laparoscopic lateral colostomy (LC) is a palliative surgery that takes a shorter time to perform; it is used in patients with unresectable rectal tumors awaiting response to adjuvant therapy or in patients with a high anesthetic risk.

- extended Hartmann operation (EHO) with total mesorectal excision or transection; first described in 1921 by the French surgeon Henri Albert Hartmann as the surgical resection of the rectosigmoid colon with closure of the rectal stump and end colostomy, used to treat cancer or rectosigmoid diverticulitis $[9,10]$. It is a curative-intent surgery by which the rectal tumor is removed with oncologic safety limits with mesorectal excision (total in mid rectal tumors or partial in the upper ones) after ligation of the inferior mesenteric vessels at the origin or above the emergence of the left colic artery. The closure of the rectal stump can be done mechanically (mid rectal cancer) or manually (upper rectal cancer). Postoperative pelvic drainage can be exteriorized abdominally (EHO-abd)or perineally (EHO-per). It is considered a sequential operation, because a second surgery in addition to restoring digestive continuity also includes an intraoperative assessement of the lesion and the surgical resolution of any local or distant recurrence. Not infrequently terminal colostomy becomes permanent. In the literature it can be found under the name of low Hartmann procedure or low anterior resection end-colostomy with closure of the distal rectal stump.

The aim of this study was to analyze the complications occurring within 60 days after surgery in patients diagnosed with upper of midrectal cancer who underwent LAR, LC or EHO.

\section{Materials and Methods}

The study is a nonrandomized, prospective analysis over a 24-month period, based on Surveillance protocol for patients with liver metastases of colorectal origin used at First Surgical Oncology Unit, Regional Institute of Oncology Iaşi, Romania. According to this protocol the patients are classified into 4 classes of hepatic resectability: resectable, possibly, susceptible, and unresectable metastases.

Inclusion criteria: age over 18 years, pathology diagnosis of rectal carcinoma, imaging diagnosis (CT/MRI) of liver metastases, rectal tumor located at more than $6 \mathrm{~cm}$ from the anal verge.

Exclusion criteria: patients diagnosed with rare rectal cancers (sarcomas, lymphomas, melanomas, endocrine tumors, carcinoid tumors), patients diagnosed with rectal tumors with anal sphincter invasion, patients who refused the treatment recommended by the Regional Institute of Oncology Commission.

Preoperative assessment included patient history, physical examination including the assessment of physical status, and anesthetic risk class according to ASA classification. Also included was the rectal examination aimed to assess anal sphincter competence useful in selecting the surgical procedure.

For the comparative evaluation of postoperative surgical complications, the selected patients were divided into 3 groups depending on the surgical procedure they underwent [11]: LAR, LC or EHO, and 2 subgroups: EHO-abd, EHO-per.

Anterior resection of the rectum (LAR) was performed in patients with ASA I/II anesthetic risk, resectable tumors (R0) without extrahepatic metastases. In mid rectal tumors total mesorectal excision, identification of hypogastric nerve, mobilization of the splenic angle of the colon, and stapled end-to-end colorectal anastomosis were performed; protective ileostomy was performed in all patients. Pelvic drainage was exteriorized abdominally. In upper rectal tumors mesorectal transection or total mesorectal excision was performed depending on the intraluminal extension of the tumor. Mobilization of the splenic angle of the colon was not required in all patients. Endto-end colorectal anastomosis was usually hand-sewn and protective ileostomy was performed only when there were doubts of anastomotic tightness. To meet all demands of radicality of surgery the following associated interventions were performed: anexectomy, hysterectomy, enterectomy, partial cystectomy, and appendectomy. Liver metastases were classified as resectable or possibly and we chose to perform metastasectomies (atypical limited liver resections or wedge resections), thermoablation and/or insertion of port-a-cath in the hepatic artery.

The indication for performing lateral colostomy (LC) was: unresectable rectal tumor, unresectable liver and extrahepatic metastases, or ASA III/IV anesthetic risk. Liver metastases were classified into the following resectability classes: possibly, susceptible or unresectable.

Extended Hartmann operation (EHO) was performed in patients at ASA II/ or III anesthetic risk, patients in whom after rectal tumor removal there was a suspicion, biopsy-confirmed or unconfirmed of residual pelvic tumor, with or without extrahepatic metastases. Total mesorectal excision was required in mid rectal tumors when the stapled closure of the rectal stump and pelvic drainage exteriorized abdominally or perineal pararectal were practiced. In the upper rectal tumors, mesorectal transection and closure of the rectal stump were performed either by using stapler (pelvic drainage abdominally exteriotized) or hand-sewn (pelvic drainage transrectal exteriorized). The associated surgical interventions are the same as in the curativeintend procedures: ovariectomy, hysterectomy, enterectomy, partial cystectomy, appendectomy and peritoneal biopsies - most frequently.

Data were extracted from IRO Iasi electronic system and observation sheets. The general characteristics of the patients (age, gender) were examined. The associated diseases were analyzed and classified using of ASA Risk Score and Charlson Comorbidity Index Risk [12]. Postoperative surgical complications (postoperative wound complications, stoma complications, postoperative bleeding, pelvic abscess, prolonged postoperative ileus, anastomotic leakag, reinterventions, readmissions, postoperative mortality) were analyzed according to Dindo-Clavien Classification.

The obtained data were processed in MS Excel, and statistical analysis was performed with RStudio software. Fisher exact test of independence was used. Significance threshold was $\mathrm{p}<0.05$.

\section{Results}

In the interval June 2012 - May 2014, at IRO Iasi, Romania, 87 patients were diagnosed with upper and mid rectal cancer with liver metastases; LAR was performed in 18 patients, LC in 19 patients, and 50 patients underwent EHO with pelvic drainage abdominally exteriorized ( $n=37$, subgroup EHO-abd) or perineally ( $n=13$, subgroup EHO-per) (Figure 1,4,5).

The general characteristics of patients (age, sex), comorbidities (according to ASA Risk Score and Charlson Comorbidity Index) and primary tumor staging according to pathological analysis are shown in Table 1, Figure 2 and Figure 3.

As expected, we found a significant association $\left(\mathrm{p}<10^{-10}\right)$ between the type of surgery and anesthetic risk expressed by ASA score. This can be explained by the careful selection of patients according to the extent and risks of surgery. Survival expressed by Charlson Comorbidity Index also showed a significant association $\left(\mathrm{p}<10^{-7}\right)$ with the type of surgery.

Postoperative surgical complications are presented in detail, before and after the use of Dindo-Clavien Classification, in Table 2 and 3. 


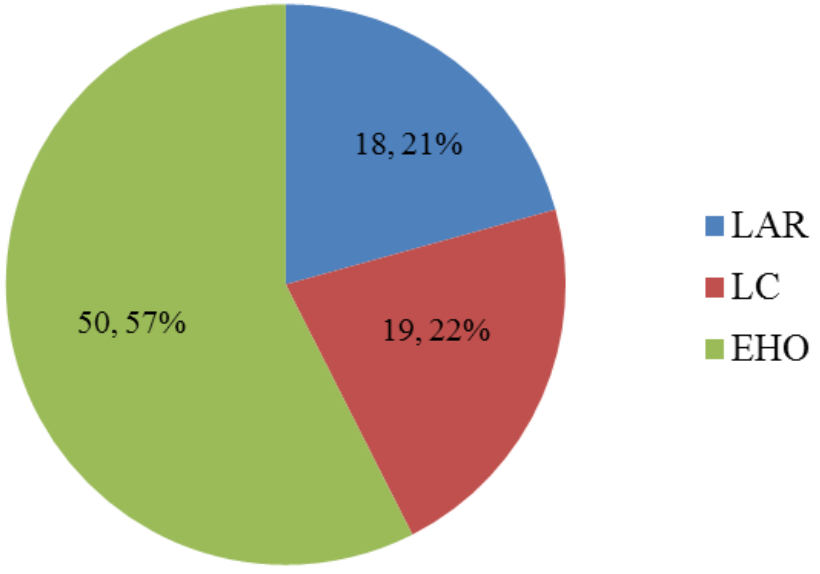

Figure 1: Percentage distribution for each type of surgery

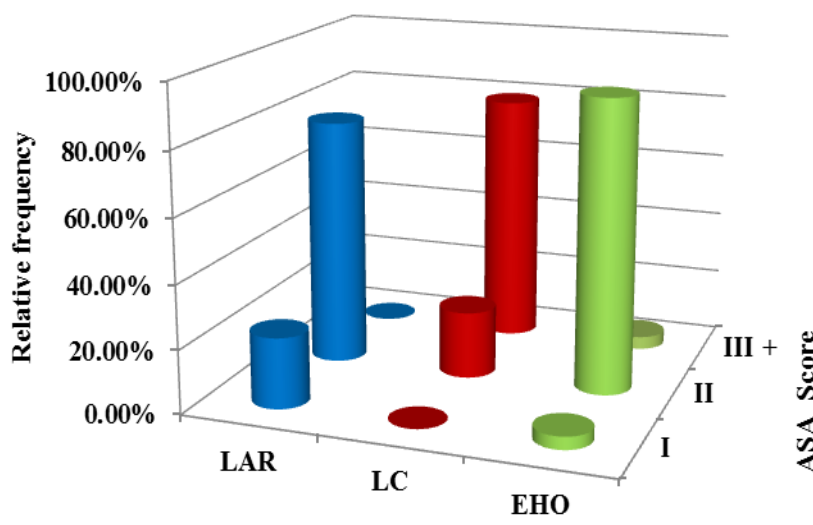

Surgical procedure

Figure 2: Relative frequency of the anesthetic risk expressed by ASA score for each type of surgery

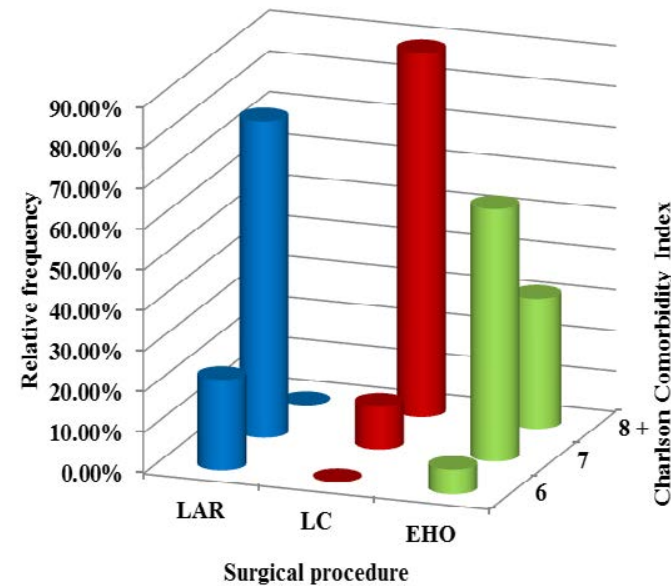

Figure 3: Relative frequency of the survival prognostic expressed by Charlson Comorbidity Index for each type of surgery

Surgical wound complications (seromas, hematomas, infections, dehiscence) were more common among the patients who underwent LC (36.84\%) compared with those who underwent LAR and EHO with showed approximately equal rates (22.22\% vs. $22 \%)$. No ileostomy complications were recorded in the patients who underwent LAR, but a relatively high rate of colostomy complications were found in patients who underwent LC (15.78\%) compared with those who underwent EHO (8\%). Postoperative bleeding did not require reinterventions. In percentages, there was a important difference between postoperative bleeding in patients with LAR and those with EHO (16.66\% vs. 6\%). A higher rate of pelvic abscesses was found in patients who underwent EHO $(24 \%, n=12)$ of which 9 patients $(18 \%)$ required reinterventions for pelvic collection drainage; all these patients had pelvic drainage abdominally exteriorized ( EHO-abd). No presacral abscesses were found in patients with pelvic drainage perineally exteriorized (EHO-per).

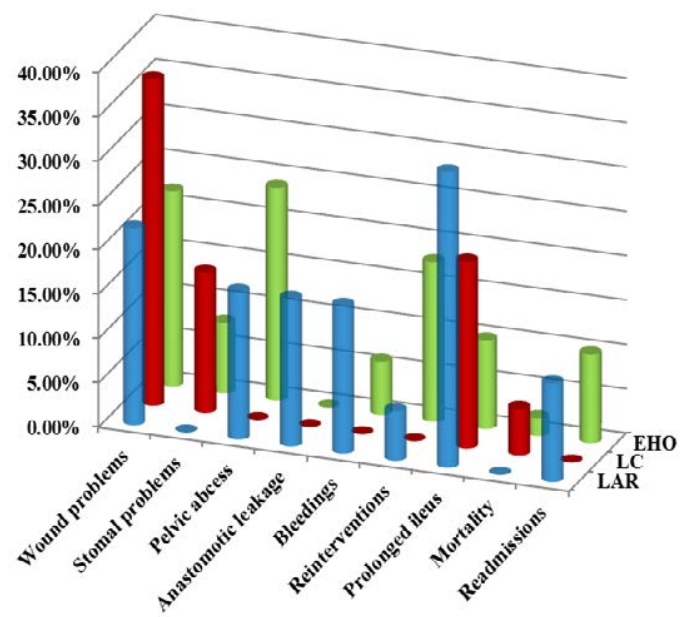

Figure 4: Distribution of the postoperative surgical complications for each type of surgery

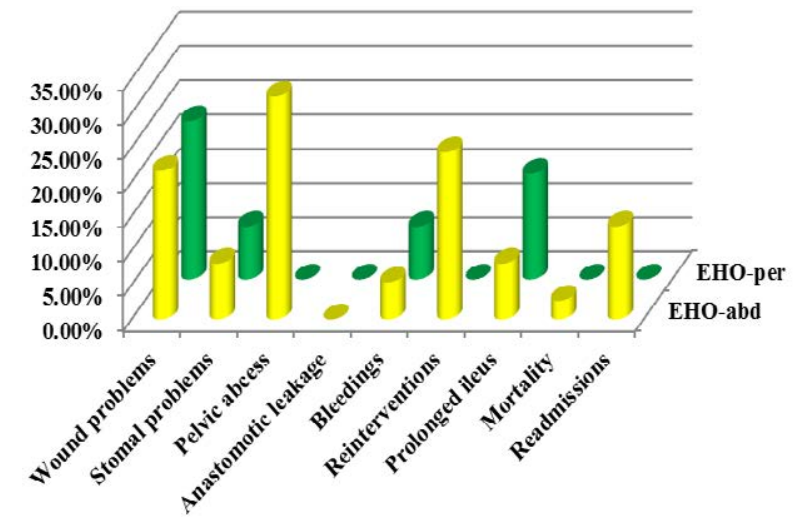

Figure 5: Distribution of the postoperative surgical complications for each subgroup $\mathrm{EHO}$

Table I: Patient and tumour characteristics according to type of surgical intervention

\begin{tabular}{|l|c|c|c|c|c|}
\hline Variables & LAR n=18 & LC $\mathbf{n = 1 9}$ & EHO $\mathbf{n = 5 0}$ & Total $\mathbf{n = 8 7}$ & p-value \\
\hline Age (mean) & 65.77 & 66 & 61.84 & 63.56 & - \\
\hline Gender & & & & & 0.55 \\
\hline Male & $10(55.55 \%)$ & $9(47.37 \%)$ & $31(52 \%)$ & $50(57.47 \%)$ & \\
\hline Female & $8(44.45 \%)$ & $10(52.63 \%)$ & $19(48 \%)$ & $37(42.52 \%)$ & \\
\hline ASA Score & & & & & $<\mathbf{1 0}^{-10}$ \\
\hline I & $4(22.22 \%)$ & 0 & $2(4.00 \%)$ & $6(6.90 \%)$ & \\
\hline II & $14(77.78 \%)$ & $4(21.05 \%)$ & $46(92.00 \%)$ & $64(73.56 \%)$ & \\
\hline III + & 0 & $15(78.95 \%)$ & $2(4.00 \%)$ & $17(19.54 \%)$ & \\
\hline $\begin{array}{l}\text { Charlson } \\
\text { Comorbidity } \\
\text { Index }\end{array}$ & $4(22.22 \%)$ & 0 & $3(6.00 \%)$ & $7(8.05 \%)$ & \\
\hline $\mathbf{6}$ & $14(77.78 \%)$ & $2(10.53 \%)$ & $31(62.00 \%)$ & $47(54.02 \%)$ & \\
\hline $\mathbf{7}$ & 0 & $17(89.47 \%)$ & $16(32.00 \%)$ & $33(37.93 \%)$ & \\
\hline $\mathbf{8 +}$ & & & & & - \\
\hline pT-stage & $12(66.67 \%)$ & n.a. & $26(52.00 \%)$ & & \\
\hline pT3 & $6(33.33 \%)$ & n.a. & $24(48.00 \%)$ & & \\
\hline pT4a,b & & & & \\
\hline
\end{tabular}


Table II: Postoperative surgical complications for each type of surgery

\begin{tabular}{|c|c|c|c|c|c|c|c|c|}
\hline Variables & $\begin{array}{l}\text { LAR } \\
n=18\end{array}$ & $\begin{array}{c}\text { LC } \\
n=19\end{array}$ & $\begin{array}{l}\text { EHO } \\
n=50\end{array}$ & p-value & $\begin{array}{l}\text { TOTAL } \\
\text { n=87 }\end{array}$ & $\begin{array}{c}\text { EHO-abd } \\
\qquad n=37\end{array}$ & $\begin{array}{c}\text { EHO-per } \\
n=13\end{array}$ & $\begin{array}{c}\text { p- } \\
\text { value }\end{array}$ \\
\hline Wound problems & $4(22.22 \%)$ & $7(36.84 \%)$ & $\begin{array}{c}11 \\
(22 \%)\end{array}$ & 0.43 & $22(25.29 \%)$ & $8(21.62 \%)$ & $\begin{array}{c}3 \\
(23.08 \%)\end{array}$ & 1 \\
\hline Stomal problems & 0 & $3(15.78 \%)$ & $\begin{array}{c}4 \\
(8 \%)\end{array}$ & 0.22 & $7(8.05 \%)$ & $\begin{array}{c}3 \\
(8.11 \%)\end{array}$ & $1(7.69 \%)$ & 1 \\
\hline Postoperative bleedings & $3(16.66 \%)$ & 0 & $\begin{array}{c}3 \\
(6 \%)\end{array}$ & 0.12 & $11(12.67 \%)$ & $\begin{array}{c}2 \\
(13.51 \%)\end{array}$ & $1(7.69 \%)$ & 1 \\
\hline Pelvic abscess & $3(16.66 \%)$ & 0 & $\begin{array}{c}12 \\
(24 \%)\end{array}$ & 0.041 & $\begin{array}{c}15 \\
(17.24 \%)\end{array}$ & $12(32.43 \%)$ & 0 & 0.0221 \\
\hline Anastomotic leakage & $3(16.66 \%)$ & n.a. & n.a. & - & $3(3.45 \%)$ & n.a. & n.a. & - \\
\hline Prolonged ileus & $6(33.33 \%)$ & $4(21.05 \%)$ & $\begin{array}{c}5 \\
(10 \%)\end{array}$ & 0.08 & $15(17.24 \%)$ & $\begin{array}{c}3 \\
(8.11 \%)\end{array}$ & $2(15.38 \%)$ & 0.59 \\
\hline Reinterventions & $1(5.55 \%)$ & 0 & $\begin{array}{c}9 \\
(18 \%)\end{array}$ & 0.07 & $10(11.49 \%)$ & $9(24.32 \%)$ & 0 & 0.08 \\
\hline Readmissions & $2(11.11 \%)$ & 0 & $\begin{array}{c}5 \\
(10 \%)\end{array}$ & 0.43 & $7(8.05 \%)$ & $5(13.51 \%)$ & 0 & 0.30 \\
\hline Postoperative mortality & 0 & $1(5.26 \%)$ & $\begin{array}{c}1 \\
(2 \%)\end{array}$ & 0.67 & $\begin{array}{c}2 \\
(2.30 \%)\end{array}$ & $1(2.70 \%)$ & 0 & 1 \\
\hline
\end{tabular}

Table III: Standardized surgical complications by Clavien - Dindo Classification

\begin{tabular}{|l|c|c|c|c|}
\hline The Clavien-Dindo & LAR & LC & EHO & TOTAL \\
\hline Classification & $\mathbf{n = 1 8}$ & $\mathbf{n = 1 9}$ & $\mathbf{n = 5 0}$ & $\mathbf{n = 8 7}$ \\
\hline Grade I & 4 & 9 & 14 & 27 \\
\hline Grade II & $(22.22 \%)$ & $(47.36 \%)$ & $(28 \%)$ & $(31.03 \%)$ \\
\hline \multirow{2}{*}{ Grade III } & 6 & 4 & 9 & 19 \\
\hline Grade IV & $(33.33 \%)$ & $(21.05 \%)$ & $(18 \%)$ & $(21.83 \%)$ \\
\hline & 1 & 0 & 9 & 10 \\
\hline Grade V & $(5,55 \%)$ & & $(18 \%)$ & $(11.49 \%)$ \\
\hline
\end{tabular}

Fisher's test of independence was used to each type of postoperative complication for identifying statistically significant differences between the frequency and type of surgical complication. At this stage, the study included three groups - LAR, LC and EHO - without distinguishing between drainage types in the $\mathrm{EHO}$ group. Very close to the significance threshold of 0.05 , the number of reinterventions varied considerably by procedure type $(\mathrm{p}=0.07)$, with a higher frequency for $\mathrm{EHO}$; all reinterventions in the EHO group were related to EHO-abd subgroup, no reinterventions being performed for EHO-per subgroup.

Prolonged ileus, also very close to the significance threshold $(\mathrm{p}=0.08)$, had a higher incidence in LAR and LC than in ECO.

For the other postoperative complications, we do not have statistically significant evidence for a procedure type-related dependence.

Assuming that the pelvic abscess rate tends to be $0 \%$ in LC-type procedures, the frequency differentiation of pelvic abscesses could be analyzed by applying the Fisher test to the surgical procedure types that can cause this complication (Table 2). Test result $(\mathrm{p}=0.041)$ indicated significant differences in the incidence of pelvic abscesses by type of surgical procedure. Comparing only the two EHO groups the pelvic abscess rate was significantly higher for EHO-abd compared with EHO-per $(\mathrm{p}=0.0221)$.

The $95 \%$ confidence interval for the proportion of pelvic abscesses in LAR procedures was $(4.40 \%, 42.26 \%)$, while for EHO-per it was $(0 \%$, $28.34 \%$ ) - (i.e. the probability of more than $28.34 \%$ pelvic abscess is only $5 \%$ for $\mathrm{EHO}$ with perineal drainage).

\section{Discussions}

Postoperative wound complications have proven to be a relatively numerous in all three groups (perhaps due to the proximity to the stoma), but without significant differences. All these surgical wound complications were minor (Clavien - Dindo grade I). The rate of colostomy complications was higher in the LC group, but were most commonly minor. In the EHO group one patient developed colostomy necrosis causing his death. Prolonged postoperative ileus was found in large numbers of patients in LAR group, one patient requiring hospital readmission. Appreciable differences, close to statistical significance $(\mathrm{p}=0.08)$ were found between the 3 groups.

The main complication in this study was the pelvic abscess (presacral abscess). In the LAR group pelvic abscesses occurred in patients who developed postoperative anastomotic fistulas, one patient requiring reintervention for drainage of presacral collection. The large number of pelvic abscesses $(24 \%, \mathrm{n}=12)$ in EHO group determined a high reintervention rate $(18 \%, n=9)$, some of them performed during the same hospitalization, others $(13.16 \%, n=5) 3-4$ weeks after a new admission. These results are in agreement eith those reported in the literature [13-16]. The analysis of the two EHO subgroups (EHO-abd and EHO-per) showed that all abscesses occurred in patients with drainage abdominally exteriorized. These encouraging results of the use of drainage perineally exteriorized made us consider its extended use in LAR- type surgeries. 
Drainage of abscesses in EHO group patients was performed under sedation, transrectal, under ultrasound guidance, via a laparoscopic trocar through which a drain was introduced.

A recent Dutch study [17] suggests a significant association between the amount of blood lost during LAR surgery and anastomotic fistula - with high mortality and morbidity due to pelvic abscess formation - suggesting the use of an EHO-type surgical procedure in the case of severe introperative bleeding. There are studies concluding that EHO is a lifesaving operation, but not devoid of postoperative complication risks related to colostomy and frequent occurrence of pelvic abscess, without discussing the postoperative drainage type [18]. Patients who undergo LAR may develop anal incontinence (postoperatively or post radiation therapy) affecting their quality of life [19]. In patients with colostomy, their quality of life does not appear to be affected, even if colostomy is permanent [20].

\section{Conclusions}

Extended Hartmann operation remains a safe alternative technique, which shares indications with LAR and LC, and is associated with a higher rate of pelvic abscesses and reinterventions that can be avoided by postoperative perineal drainage of the presacral space. It is a lifesaving surgery when performimg LAR fails due to intraoperative incidents.

\section{Acknowledgement}

This study received financial support through the: "Program of Excellence in multidisciplinary doctoral and postdoctoral research in chronic diseases", contract identification number: HRD / 159 / 1.5 / S / 133377. Beneficiary "Gr. T. Popa "lasi, financed from the European Social Fund Operational Programme Human Resources Development 2007-2013." Authors have no conflict of interests to disclose.

\section{References}

1. Ferlay J, Steliarova-Foucher E, Lortet-Tieulent J, Rosso S, Coebergh JW, et al. (2013) Cancer incidence and mortality patterns in Europe: estimates for 40 countries in 2012. Eur J Cancer 49: 1374-1403.

2. Heald RJ, Husband EM, Ryall RD (1982) The mesorectum in rectal cancer surgery--the clue to pelvic recurrence? Br J Surg 69: 613-616.

3. [No authors listed] (1996) Local recurrence rate in a randomised multicentre trial of preoperative radiotherapy compared with operation alone in resectable rectal carcinoma. Swedish Rectal Cancer Trial. Eur J Surg 162: 397-402.

4. Sauer R, Becker H, Hohenberger W, Rödel C, Wittekind C, et al. (2004) Preoperative versus postoperative chemoradiotherapy for rectal cancer. N Engl J Med 351: 1731-1740.
5. Vogelstein B, Fearon ER, Hamilton SR, Kern SE, Preisinger AC, et al. (1988) Genetic alterations during colorectal-tumor development. N Engl J Med 319: 525-532.

6. Fearon ER, Vogelstein B (1990) A genetic model for colorectal tumorigenesis Cell 61: 759-767.

7. Nordlinger B, Guiguet M, Vaillant JC, Balladur P, Boudjema K, et al. (1996) Surgical resection of colorectal carcinoma metastases to the liver. A prognostic scoring system to improve case selection, based on 1568 patients. Association Française de Chirurgie. Cancer 77: 1254-1262.

8. Hurwitz H, Fehrenbacher L, Novotny W, Cartwright T, Hainsworth J, et al (2004) Bevacizumab plus irinotecan, fluorouracil, and leucovorin for metastatic colorectal cancer. N Engl J Med 350: 2335-2342.

9. Desai DC, Brennan EJ Jr, Reilly JF, Smink RD Jr (1998) The utility of the Hartmann procedure. Am J Surg 175: 152-154.

10. Hartmann, H (1921) 30th Congress Francais de Chirurgie-Process. Verheaux Memoires et Discussions 30:41.

11. Dindo D, Demartines N, Clavien PA (2004) Classification of surgical complications: a new proposal with evaluation in a cohort of 6336 patients and results of a survey. Ann Surg 240: 205-213.

12. Charlson ME, Pompei P, Ales KL, MacKenzie CR (1987) A new method of classifying prognostic comorbidity in longitudinal studies: development and validation. J Chronic Dis 40: 373-383.

13. Vermeer TA, Orsini RG, Daams F, Nieuwenhuijzen GA, Rutten HJ (2014) Anastomotic leakage and presacral abscess formation after locally advanced rectal cancer surgery: Incidence, risk factors and treatment. Eur J Surg Oncol pii: S0748-7983: 00381-3.

14. Molina Rodríguez JL, Flor-Lorente B, Frasson M, García-Botello S, Esclapez P et al. (2011) Low rectal cancer: abdominoperineal resection or low Hartmann resection? A postoperative outcome analysis. Dis Colon Rectum 54: 958-962.

15. Snijders HS, Wouters MW, van Leersum NJ, Kolfschoten NE, Henneman D, et al. (2012) Meta-analysis of the risk for anastomotic leakage, the postoperative mortality caused by leakage in relation to the overall postoperative mortality. Eur J Surg Oncol 38: 1013-1019.

16. Buhre LM, Plukker JT, Mehta DM, Verschueren RC, Oldhoff J (1991) The extended Hartmann operation as an elective procedure for rectal cancer. A forgotten operation. Eur J Surg Oncol 17: 502-506.

17. Bakker IS, Snijders HS, Wouters MW, Havenga K, Tollenaar RA, et al. (2014) High complication rate after low anterior resection for mid and high rectal cancer; results of a population-based study. Eur J Surg Oncol 40: 692-698.

18. Tøttrup A, Frost $L$ (2005) Pelvic sepsis after extended Hartmann's procedure. Dis Colon Rectum 48: 251-255.

19. Rasmussen OO, Petersen IK, Christiansen J (2003) Anorectal function following low anterior resection. Colorectal Dis 5: 258-261.

20. Pachler J, Wille-Jorgensen $P$ (2012) Quality of life after rectal resection for cancer, with or without permanent colostomy. Cochrane Database Syst Rev12:CD004323. 